\title{
Quantitative Analysis of Major and Minor Elements in Lead-free Solder Chip by LA-ICP-MS
}

\author{
Koyo Ido, ${ }^{* 1, * 2}$ Hideyuki ObaYaSHI, ${ }^{* 3, * 4}$ Yanbei ZhU, ${ }^{* 1 \dagger}$ Takafumi Hirata, ${ }^{* 3}$ Akiko Hokura, $* 2$ \\ Naoko Nonose, $* 1$ and Kazumi INAGAKI $* 1, * 2$ \\ *1 National Metrology Institute of Japan (NMIJ), National Institute of Advanced Industrial Science and \\ Technology (AIST), 1-1-1 Umezono, Tsukuba, Ibaraki 305-8563, Japan \\ *2 Graduate School of Engineering, Tokyo Denki University, 5 Senju Asahi-cho, Adachi, Tokyo 120-8551, Japan \\ *3 Geochemical Research Center, The University of Tokyo, 7-3-1 Hongo, Bunkyo, Tokyo 113-0033, Japan \\ *4 Laboratory for Planetary Sciences, Kyoto University, Kitashirakawa Oiwakecho, Sakyo, Kyoto 600-8502, Japan
}

\begin{abstract}
A method was established for the quantitative analysis of the elements $(\mathrm{Cu}, \mathrm{Ag}, \mathrm{Pb}$, and $\mathrm{Sn})$ in solder samples by laser ablation inductively coupled plasma mass spectrometry (LA-ICP-MS), with Sn-based matrix matched standard solutions for defining the calibration curves. It was found that chloride-ion presented in commercially available $\mathrm{Sn}$ standard solution resulted in a precipitation of $\mathrm{AgCl}$ and caused the deterioration of the linearity of the calibration curve for $\mathrm{Ag}$. Therefore, a laboratory-made chloride-free $\mathrm{Sn}$ solution was used to prepare $\mathrm{Sn}$ matrix matched standard solutions so as to ensure the stability of the elements including Ag. For the quantitative analysis of solder samples by LA-ICP-MS, the operating conditions of the LA instruments were set to obtain a fluence of over $12 \mathrm{~J} \mathrm{~cm}^{-2}$. This is mainly because of larger LA-induced elemental fractionations using a fluence of $<10 \mathrm{~J} \mathrm{~cm}^{-2}$. The results for $\mathrm{Ag}, \mathrm{Cu}, \mathrm{Pb}$, and $\mathrm{Sn}$ in a certified reference material (NMIJ CRM 8203-a) were close to, or in agreement with, the certified values, indicating that the present method was valid for the quantitative analysis of the elements in solder samples. In comparison to the certified values, relatively larger uncertainties were obtained for the analytical results by LA-ICP-MS, which could be attributed to the dependence on the homogeneity of the sample because the sample aliquots used for analysis were much smaller than those required for the traditional analytical procedures (i.e., sample quantity ratio of ca. 1:13000). Further improvement of the uncertainty might be obtained by using a larger sample quantity for the analysis by LA-ICP-MS so as to improve the representativeness of the sample.
\end{abstract}

Keywords LA-ICP-MS, fractionation, quantitative analysis, solder, matrix match

(Received January 31, 2018; Accepted March 27, 2018; Published June 10, 2018)

\section{Introduction}

Laser ablation inductively coupled plasma mass spectrometry (LA-ICP-MS) has been widely studied as an analytical technique for the rapid analysis of solid samples without transforming the sample to a solution. Applications of LA-ICP-MS have been reported in geological, environmental, biological, archaeological and medicine samples, respectively. ${ }^{1-10}$ Direct analysis of solid samples by using LA-ICP-MS permits high-throughput analysis compared to those transforming the sample to a solution. Various studies had been carried out particularly on developing approaches for quantitation by using LA-ICP-MS. ${ }^{11-16}$ The reason is that the calibration strategy is often challenging for reliable quantitative analysis due to LA-induced elemental fractionation and matrix effects in a measurement by ICPMS. ${ }^{16-20}$

Optimization of the operating conditions for laser ablation could suppress the elemental fractionation, while the application of a matrix matched standard should help to cancel the matrix

$\dagger$ To whom correspondence should be addressed.

E-mail: yb-zhu@aist.go.jp effect in ICP-MS. Therefore, solid standards having almost the same matrix composition as a sample are preferred. However, it is difficult to obtain matrix matched solid standards (reference materials) for most practical samples.

In recent years, calibration with a standard solution had been investigated and verified as an alternative approach to solid standards used in LA-ICP-MS. As calibration methods using standard solutions, introducing the standard solution using desolvation and ultrasonic nebulization techniques have been reported, where the solution mist generated by a nebulizer was mixed just before the ICP torch. ${ }^{21-27}$ In some of these works, an addition apparatus such as a desolvation unit were required in addition to the default parts of the LA instrument and ICPMS instrument. Such an additional apparatus might cause a longer wash-out time due the memory-effect. As an alternative approach, the present authors had reported a method using a matrix-matched standard solution for quantitative analysis of trace elements in glass, ${ }^{28}$ in which the sample aerosol from a makeup gas port was mixed with the solution mist generated by a nebulizer in the spray chamber, and then introduced into the ICP torch. In comparison to the conventional method, it has a superior signal stability and a simplified structure.

In the present work, the authors further investigated this 
Table 1 LA-system operating conditions

\begin{tabular}{lll}
\hline \multicolumn{1}{c}{$\begin{array}{c}\text { ESI } \\
\text { NWR213 }\end{array}$} & \multicolumn{1}{c}{$\begin{array}{c}\text { CyberLaser } \\
\text { IFRIT Type-C }\end{array}$} \\
\hline Laser type & Nd:YAG nanosecond & Ti:S femtosecond \\
Wavelength & $213 \mathrm{~nm}(5 \mathrm{HG})$ & $260 \mathrm{~nm}(\mathrm{THG})$ \\
Fluence & $24 \mathrm{~J} \mathrm{~cm}^{-2}$ & $11.3 \mathrm{~J} \mathrm{~cm}^{-2}$ \\
Frequency & $10 \mathrm{~Hz}$ & $10 \mathrm{~Hz}$ \\
Spot size & $10 \mu \mathrm{m}$ & $15 \mu \mathrm{m}$ \\
Scan speed & $100 \mu \mathrm{m} \mathrm{s}^{-1}$ & $>1 \mathrm{~mm} \mathrm{~s}{ }^{-1}$ \\
Carrier gas & $\mathrm{He}, 0.8 \mathrm{~L} \mathrm{~min}^{-1}$ & $\mathrm{He} 0.6, \mathrm{Ar} 0.5 \mathrm{~L} \mathrm{~min}^{-1}$ \\
\hline
\end{tabular}

Table 2 ICP-MS operating conditions

\begin{tabular}{lll}
\hline & Agilent 8800 & $\begin{array}{c}\text { Thermo Fisher } \\
\text { Scientific iCap Q }\end{array}$ \\
\hline RF Power & $1500 \mathrm{~W}$ & $1550 \mathrm{~W}$ \\
Carrier gas & $0.60 \mathrm{~L} \mathrm{~min}^{-1}$ & $0.55 \mathrm{~L} \mathrm{~min}^{-1}$ \\
Cell gas & $\mathrm{H}_{2}, 3 \mathrm{~mL} \mathrm{~min}^{-1}$ & - \\
MS/MS operation & On-mass & Single mass \\
Measurement mode & TRA & TRA \\
Integrate time & $0.01 \mathrm{~s}$ & $0.01 \mathrm{~s}$ \\
\hline
\end{tabular}

method as an approach for the quantitative analysis of $\mathrm{Ag}, \mathrm{Cu}$, and $\mathrm{Pb}$ in lead-free solder samples, while taking into consideration the fact that $\mathrm{Pb}$ concentration is restrictively regulated by the Restriction on Hazardous Substances (RoHS). From the viewpoint that one of the essential requirements for the present method is the matrix matched standard solution for calibration, special attention was paid to the assurance for the stability of the elemental concentration in matrix matched standard solutions. On the other hand, LA-induced elemental fractionation is often more troublesome for the measurement of metal samples due to their good thermo-conductivity in comparison to other matrix such as glasses. Optimization of the LA operating conditions was carried out in the present work so as to achieve the best suppression of LA-induced elemental fractionation. Furthermore, the performances of nanosecond (ns) and femtosecond (fs) laser ablation systems were investigate to compare the difference in LA-induced elemental fractionation. Validation of method was carried out by analyzing a certified reference material (CRM) for the analysis of major and minor elements in lead-free solder.

\section{Experimental}

\section{Instrumentation}

The ns-LA-ICP-MS system consisted of an ICP-MS instrument (Agilent8800, Agilent Technologies Japan, Tokyo, Japan) and a ns-LA instrument (NWR213, Elemental Scientific, Inc. Japan, Tokyo, Japan). The fs-LA-ICP-MS system consisted of an ICP-MS instrument (iCAP Q-c, Thermo Fisher Scientific, Waltham, USA) and an fs-LA instrument (IFRIT Type-C, Cyber Laser Inc., Tokyo, Japan). The operating conditions for the LA instruments and ICP-MS instruments are summarized in Tables 1 and 2, respectively, where the parameters were optimized daily to obtain the best sensitivity for analysis. The sample aerosol generated by the LA system was introduced into the spray chamber of the ICP-MS through the makeup gas port, where He was used as the carrier gas for the LA system. In the

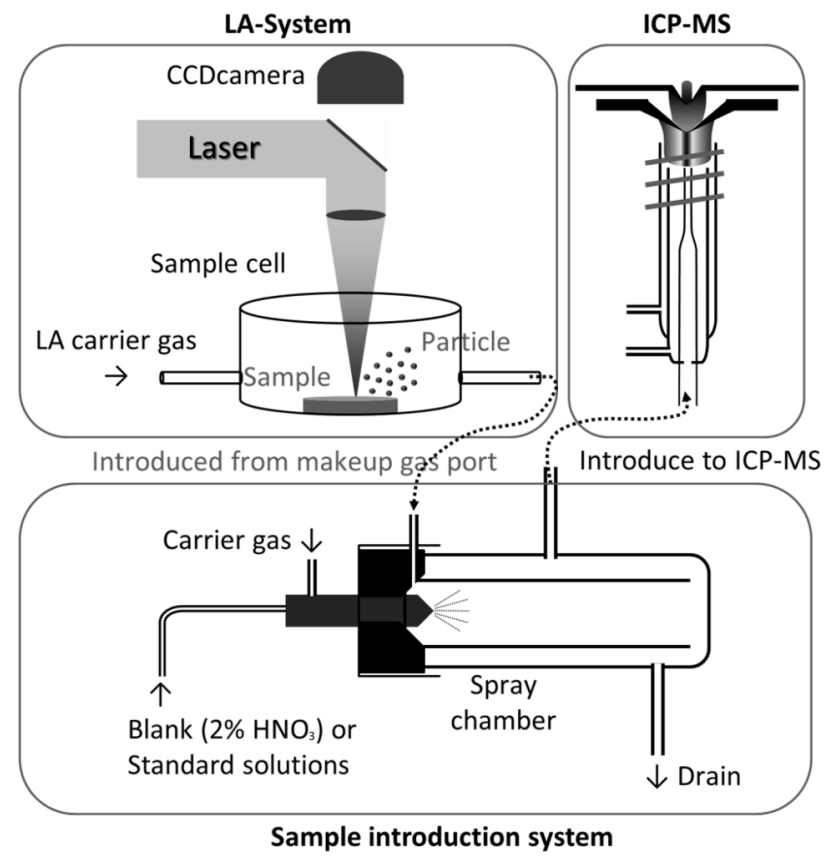

Fig. 1 Schematic diagram of the sample introduction system for the LA-ICP-MS.

spray chamber, the sample aerosol was mixed with the solution mist generated by a nebulizer and then introduced into the ICP (Fig. 1). In the present method, default parts of the ICP-MS instruments and the LA systems were sufficient for constructing the systems, without an additional apparatus, such as a desolvation instrument.

\section{Chemicals and materials}

In order to ensure the metrological traceability of the results, single-element standard solutions $\left(1000 \mathrm{mg} \mathrm{L}^{-1}\right.$, guaranteed by the Japan Calibration Service System, JCSS) of $\mathrm{Ag}, \mathrm{Cu}, \mathrm{Pb}, \mathrm{Sn}$ were purchased from Kanto Chemical Co., Inc. (Tokyo, Japan). Furthermore, preparation of the calibrating solutions was carried out using an electronical balance calibrated by JCSS. Ultrapure ${ }^{\circledR}$ grade $\mathrm{HNO}_{3}$ and $\mathrm{HF}$ for making standard solutions was also purchased from Kanto Chemical Co., Inc. High-purity tin (shot 99.9\%) was purchased from Wako Pure Chemical Industries, Ltd. (Osaka, Japan) and used for the preparation of a standard solution. Pure water used throughout the present experiment was prepared using a Millipore purification system (Elix, Nihon Millipore Kogyo, Tokyo, Japan). A lead-free solder CRM (NMIJ CRM 8203-a Lead-Free Solder Chip-Pb High Concentration) was randomly drawn from the CRM stocks and analyzed to confirm the usefulness of the present method.

\section{Sample preparation}

Taking into consideration the property of the solder sample, Sn was used as the matrix of standard solutions and internal standard. For matrix matching, standard solutions for making a calibration curve were prepared so as to obtain at elemental concentration ratios close to those in the solder sample. Typical elemental concentrations in the sample and the standards were as follows: sample $(96.5 \% \mathrm{Sn}, 3 \% \mathrm{Ag}, 0.5 \% \mathrm{Cu}, 0.1 \% \mathrm{~Pb})$, standard solutions (1000 $\mathrm{ng} \mathrm{g}^{-1} \mathrm{Sn} ; 15,30$ and $60 \mathrm{ng} \mathrm{g}^{-1} \mathrm{Ag}$; 2.5, 5 and $10 \mathrm{ng} \mathrm{g}^{-1} \mathrm{Cu} ; 0.5,1$ and $2 \mathrm{ng} \mathrm{g}^{-1} \mathrm{~Pb}$, respectively). All the solutions were finally prepared in $0.3 \mathrm{~mol} \mathrm{~L}^{-1} \mathrm{HNO}_{3}$.

A laboratory-made Sn standard solution $\left(1000 \mu \mathrm{g} \mathrm{g}^{-1}\right)$ was 
Table 3 Parameters used in Eqs. (1) to (6)

\begin{tabular}{clc}
\hline Parameter & \multicolumn{1}{c}{ Meaning } & Unit \\
\hline$[\mathrm{M}]$ & Concentration of element M in the solid sample & $\mu \mathrm{g} \mathrm{g}^{-1}$ \\
{$[\mathrm{Sn}]$} & Concentration of $\mathrm{Sn}$ in the solid sample & $\mu \mathrm{g} \mathrm{g}^{-1}$ \\
$S_{\mathrm{M}}$ & Signal intensity of element M in the solid sample & $\mathrm{s}^{-1}(\mathrm{cps})$ \\
$S_{\mathrm{Sn}}$ & Signal intensity of $\mathrm{Sn}$ in the solid sample & $\mathrm{s}^{-1}(\mathrm{cps})$ \\
$f_{\mathrm{M}}$ & Function for the correlation of the concentration & - \\
& ratios of element $\mathrm{M}$ to $\mathrm{Sn}$ and the signal \\
& intensity ratios obtained by the ICP-MS &
\end{tabular}

prepared by the acid digestion of $0.1 \mathrm{~g}$ high-purity tin with $2 \mathrm{~mL} \mathrm{HF} \mathrm{(30 \% )} \mathrm{and} 3 \mathrm{~mL} \mathrm{HNO}_{3}(60 \%)$ at room temperature for $5 \mathrm{~min}$. This laboratory-made $\mathrm{Sn}$ standard solution was properly diluted to obtain the calibration standard solutions in $0.3 \mathrm{~mol} \mathrm{~L}^{-1} \mathrm{HNO}_{3}$. The concentration of $\mathrm{Sn}$ in the laboratorymade Sn standard solution was quantified by an inductively coupled plasma optical emission spectrometer using calibration standard solutions covering multiple Sn concentration levels, which were made from a JCSS-guaranteed and SI-traceable Sn standard solution. The present laboratory-made Sn standard solution was stable for at least over 3-month, which could be attributed to the formation of stable fluoro-complex of Sn during acid digestion.

\section{Calculation for quantitative analysis by LA-ICP-MS}

All of the parameters used in Eqs. (1) to (6) are summarized in Table 3.

Taking into consideration the fact that solder samples are alloys of $\mathrm{Sn}$ with $\mathrm{Ag}$ and $\mathrm{Cu}$, the sum of the elemental concentrations in a solder sample is one, which could be expressed with

$$
\Sigma[\mathrm{M}]=1 .
$$

Furthermore, Eq. (2) could be obtained by dividing both sides of Eq. (1) with the concentration of Sn:

$$
\Sigma\left(\frac{[\mathrm{M}]}{[\mathrm{Sn}]}\right)=\frac{1}{[\mathrm{Sn}]} .
$$

Therefore, the concentration of Sn could be calculated from Eq. (3), which could be obtained by rearrangement of Eq. (2):

$$
[\mathrm{Sn}]=\frac{1}{\sum\left(\frac{[\mathrm{M}]}{[\mathrm{Sn}]}\right)} .
$$

Based on the correlation of the concentration ratios in the standard solutions and the signal intensity ratios obtained by the ICP-MS, the concentration ratio of element $\mathrm{M}$ and $\mathrm{Sn}$ could be calculated from

$$
\frac{[\mathrm{M}]}{[\mathrm{Sn}]}=f_{\mathrm{M}}\left(\frac{S_{\mathrm{M}}}{S_{\mathrm{Sn}}}\right)
$$

As a result, the concentration of Sn could be calculated based on Eq. (5), which could be obtained by combining Eqs. (3) and (4):

$$
[\mathrm{Sn}]=\frac{1}{\sum\left(f_{\mathrm{M}}\left(\frac{S_{\mathrm{M}}}{S_{\mathrm{Sn}}}\right)\right)} .
$$

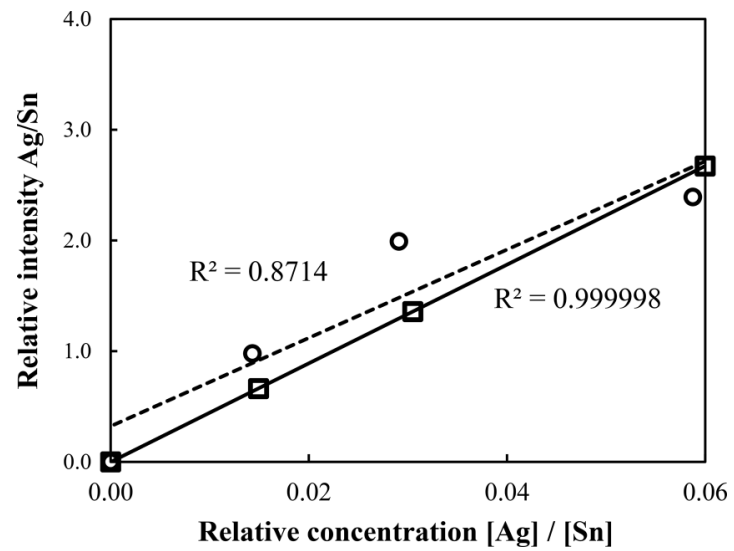

Fig. 2 Calibration curves for $\mathrm{Ag}$ defined by the Sn-based matrix matched standard solutions. Calibration curves based on commercially available Sn standard solution $(\bigcirc)$ and on laboratory-made $\mathrm{Cl}$-free $\mathrm{Sn}$ solution ( $\square$ ). Standard solutions: $1000 \mathrm{ng} \mathrm{g}^{-1} \mathrm{Sn}$; 0, 15, 30 and $60 \mathrm{ng} \mathrm{g}^{-1} \mathrm{Ag}$, respectively.

Furthermore, the concentration of element $\mathrm{M}$ could be calculated from Eq. (6), which could be obtained as the transformation of Eq. (4):

$$
[\mathrm{M}]=f_{\mathrm{M}}\left(\frac{S_{\mathrm{M}}}{S_{\mathrm{Sn}}}\right) \times[\mathrm{Sn}] .
$$

\section{Results and Discussion}

Ensuring the linearity of calibration curve for Ag in standard solutions with Sn matrix

In the present work, standard solutions were prepared with relatively higher concentration of Sn to match the matrix in the solder sample. In a preliminary experiment, the present authors found that the linearity of the calibration curve for $\mathrm{Ag}$ at higher concentration deteriorated significantly, as shown in Fig. 2, while the Ag standard solution was mixed with the commercial Sn standard solution to make mix standard solutions for establishing a calibration curve. The present authors speculated that such deterioration of the linearity of the calibration curve for $\mathrm{Ag}$ could be attributed to the formation of a $\mathrm{AgCl}$ precipitate, taking into consideration the fact that the commercially available JCSS guaranteed Sn standard solution was made in a hydrochloric acid matrix.

In order to ensure the linearity of the calibration curve for $\mathrm{Ag}$, a laboratory-made chloride-free $\mathrm{Sn}$ standard solution was obtained and used in the present work. As shown in Fig. 2, a calibration curve with good linearity (correlation coefficient, $R^{2}>0.9999$ ) was obtained for $\mathrm{Ag}$ when the laboratory-made $\mathrm{Sn}$ standard solution was applied to the preparation of $\mathrm{Ag}$ standard solutions. These results confirmed the above-mentioned speculation that the deterioration of linearity of the calibration curve for $\mathrm{Ag}$ could be attributed to the formation of a $\mathrm{AgCl}$ precipitate. It is notable that the upper range of the $x$-axis given in Fig. 2 was 0.06 , which could cover the whole concentration range of $\mathrm{Ag}$ in the present sample. A much higher concentration of $\mathrm{Ag}$ in the Sn-matrix matched standard solution could be permitted by taking into consideration the fact that a laboratorymade chlorine-free Sn solution was used in the present work.

The concentration of $\mathrm{Sn}$ in the standard solutions used in the 

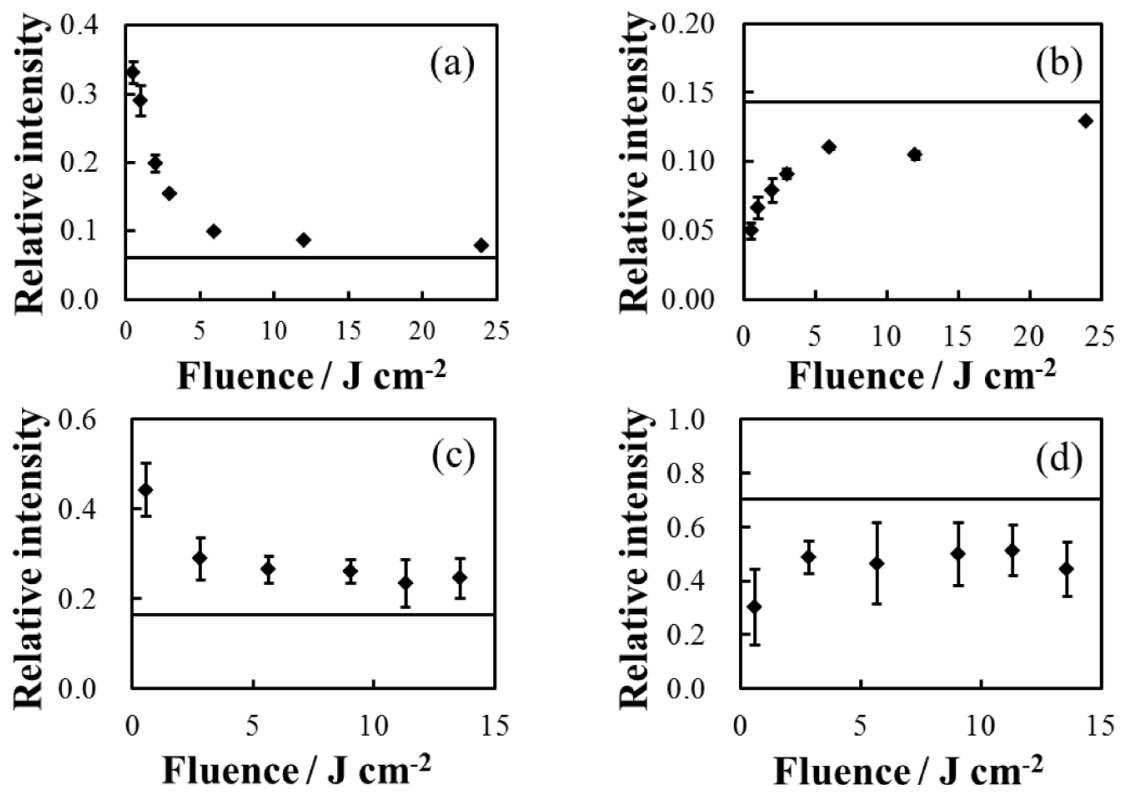

Fig. 3 Effect of laser power onto the measured $\mathrm{Pb} / \mathrm{Sn}$ ratios. Straight line, signal intensity ratio corresponding to the elemental concentrations in the standard solution. Bar, expanded uncertainty, $k=$ 2. (a) $\mathrm{Pb} / \mathrm{Sn}$ signal intensity ratio obtained by ns-LA-ICP-MS, (b) Ag/Sn signal intensity ratio obtained by ns-LA-ICP-MS, (c) Pb/Sn signal intensity ratio obtained by fs-LA-ICP-MS, (d) Ag/Sn signal intensity ratio obtained by fs-LA-ICP-MS.

present work was approximately $1000 \mathrm{ng} \mathrm{g}^{-1}$. The background equivalent concentrations (BECs), calculated based on the signal intensities in Standard-0 (1 $\mu \mathrm{g} \mathrm{g}^{-1} \mathrm{Sn}$ as the matrix, but without the addition of other elements), for $\mathrm{Cu}, \mathrm{Ag}$, and $\mathrm{Pb}$ in these standard solutions were $0.22,0.016$, and $0.006 \mathrm{ng} \mathrm{g}^{-1}$, respectively. These BECs equivalent to the concentration of $0.22,0.016$, and $0.006 \mathrm{mg} \mathrm{g}^{-1}$, respectively, in the solder sample. The detection limits (DLs), calculated based on 3-fold of the standard deviation of the signal intensities in Standard-0, for $\mathrm{Cu}$, $\mathrm{Ag}$, and $\mathrm{Pb}$ in these standard solutions were $0.12,0.013$, and $0.0011 \mathrm{ng} \mathrm{g}^{-1}$, respectively. These DLs equivalent to the concentration of $0.12,0.013$, and $0.0011 \mathrm{mg} \mathrm{g}^{-1}$, respectively, in the solder sample. All the values of BECs and DLs were low enough for the measurements in the present work.

\section{Optimization of operating conditions for LA instrument}

Quantitative analysis of the elements in a solid sample by LAICP-MS requires that the sample aliquot transported into the ICP-MS represents the chemical composition of the initial sample. Therefore, optimization of the operating conditions for the LA instrument were carried out in the present work so as to achieve the best suppression of LA-induced elemental fractionation. It had been reported that the fs-LA instrument offered an advantage of a shorter pulse duration, ${ }^{18}$ which benefited for a shorter heat diffusion time and a suppression of thermal effects. Therefore, fs-LA might suppress the LAinduced elemental fractionation in comparison to ns-LA instrument. In the present work, both the ns-LA instrument and the fs-LA instrument had been used to investigate the difference in LA-induced elemental fractionation.

In order to achieve stable and continuous signals for the elements, both the ns-LA instrument and the fs-LA instrument were operated at a frequency of $10 \mathrm{~Hz}$. At the same time, the spot size for analysis was fixed to 10 and $15 \mu \mathrm{m}$ for the ns-LA instrument and fs-LA instrument, respectively, so as to obtain sufficient signal intensity for each elements of interest.
The dependence of $\mathrm{Pb} / \mathrm{Sn}, \mathrm{Ag} / \mathrm{Sn}$, and $\mathrm{Cu} / \mathrm{Sn}$ ratio on the intensity of the laser power (i.e. fluence) on the sample surface was investigated as indicators of elemental fractionation. The results for $\mathrm{Pb} / \mathrm{Sn}$ and $\mathrm{Ag} / \mathrm{Sn}$ are plotted in Fig. 3, while the results for $\mathrm{Cu} / \mathrm{Sn}$ were similar to those for $\mathrm{Ag} / \mathrm{Sn}$, and were not plotted. It can be seen that apparently high $\mathrm{Pb} / \mathrm{Sn}$ ratio values were obtained at relatively lower laser power for both the nsLA-ICP-MS and the fs-LA-ICP-MS. The ratio of $\mathrm{Pb} / \mathrm{Sn}$ decreased gradually when the laser power increased from lower value to $10 \mathrm{~J} \mathrm{~cm}^{-2}$. While the laser power was over $10 \mathrm{~J} \mathrm{~cm}^{-2}$, a stable $\mathrm{Pb} / \mathrm{Sn}$ ratio could be obtained. It is notable that the stable $\mathrm{Pb} / \mathrm{Sn}$ ratio obtained at a laser power over $10 \mathrm{~J} \mathrm{~cm}^{-2}$ was generally in agreement with the ratio corresponding to the nominal concentration in the standard solution, suggesting that elemental fractionation was effectively suppressed. In contrast, a relatively lower value of $\mathrm{Ag} / \mathrm{Sn}$ ratio was obtained at a lower laser power, and a relatively stable value could be obtained at a fluence of over $10 \mathrm{~J} \mathrm{~cm}^{-2}$. Elemental fractionation at a relatively lower laser power could be attributed to the extremely high thermal conductivity of metallic materials, such as solder. The present results showed that a laser power providing a fluence of over $10 \mathrm{~J} \mathrm{~cm}^{-2}$ was required to suppress the elemental fraction in the laser ablation of the solder sample for quantitative analysis of the elements.

The trends of elemental fractionation for $\mathrm{Pb} / \mathrm{Sn}$ and $\mathrm{Ag} / \mathrm{Sn}$ at lower laser power were opposite to each other, i.e., the signal intensity of $\mathrm{Pb}$ was relatively higher than the predicted value of $\mathrm{Sn}$, while the signal intensity of Ag was relatively lower than the predicted value. The results could be attributed to the fact that the melting point of $\mathrm{Pb}\left(c a .330^{\circ} \mathrm{C}\right)$ was relatively close to that of $\mathrm{Sn}\left(\mathrm{ca} .230^{\circ} \mathrm{C}\right)$, while the evaporating point of $\mathrm{Pb}\left(\mathrm{ca} .720^{\circ} \mathrm{C}\right)$ was much lower than that of $\operatorname{Sn}\left(c a .1200^{\circ} \mathrm{C}\right)$. In contrast, the melting point of $\mathrm{Ag}\left(\mathrm{ca} .960^{\circ} \mathrm{C}\right)$ was much higher than that of Sn.

It is notable that the LA-induced elemental fractionation found at a lower laser power condition obtained by the ns-LA system 
(over 3-fold for $\mathrm{Pb} / \mathrm{Sn}$ signal intensity ratio) were more serious than those obtained by the fs-LA system (around 2-fold for $\mathrm{Pb} / \mathrm{Sn}$ signal intensity ratio), which could be attributed to the fact that the former provided a lower energy density per unit time (i.e., irradiance), while the latter provide a much higher energy density per unit time. However, the result obtained by fs-LA-ICP-MS and that by ns-LA-ICP-MS were comparable for each element when both systems were operated at a laser power providing a fluence over $10 \mathrm{~J} \mathrm{~cm}^{-2}$, which effectively suppressed the elemental fraction in laser ablation of solder sample.

In the present work, in order to ensure the suppression of elemental fractionation for the quantitative analysis of the elements in solder samples, laser ablation was made under a fluence of $>12 \mathrm{~J} \mathrm{~cm}^{-2}$. In order to reduce heat accumulation in the sample, a relatively higher scan speed was applied to the ns-LA instrument, while multi-spot laser ablation using galvanometric optics ${ }^{29}$ was used for the fs-LA instrument. It can be seen in Fig. 3 that the uncertainties for the results obtained by the fs-LA system were apparently higher than those obtained by the ns-LA system. One of the reasons might be that the sampling for the fs-LA system was carried out with galvanometric optics, for which a relatively larger sampling area was used for each data to calculate the uncertainty. Therefore, the block-to-block heterogeneity of an element in a relatively wider area might result in a larger uncertainty. In contrast, sampling for the ns-LA system was carried out in a relatively narrower and continuous area, for which the heterogeneity of an element in a relatively wider area might be insignificant

\section{Evaluation of homogeneity of solder sample}

In order to evaluate the heterogeneity of the solder sample, straight lines connecting the two ends of each solder surface (length: $c a .10 \mathrm{~mm}$ ) were analyzed. Profiles of the results are shown in Fig. 4. The relative standard deviation (RSD, \%) of the measurements of three different solders were as follows: $\mathrm{Cu}(24.2,14.1,16.0), \mathrm{Ag}(8.5,4.7,8.1)$ and $\mathrm{Pb}(2.7,2.1,3.1)$. Apparent heterogeneity was observed for $\mathrm{Cu}$ and $\mathrm{Ag}$ in the solder sample, while $\mathrm{Pb}$ was found with relatively good homogeneity. Based on these results, it is apparently that the homogeneity of the elements in the sample affect the results obtained by LA-ICP-MS, taking into consideration the fact that only an aliquot of the sample was ablated and transported into the ICP-MS instrument. Therefore, a relatively larger sample quantity is preferred for the analysis by LA-ICP-MS so as to cover the homogeneity of the sample.

Analytical results of major and minor element in NMIJ CRM 8203-a

In order to compensate the heterogeneity of the sample, a square range of $(190 \times 220 \mu \mathrm{m})$ was ablated for each measurement, as shown in the Fig. 5. The average value of 10 repeated measurements was considered to calculate a value for quantitative analysis. Typical results obtained by the ns-LAICP-MS are representatively summarized in Table 4. The elemental concentrations for the standard solutions and the solder sample are summarized in Table 5. It is notable that the measurements of each element in the standard solutions were generally obtained with good reproducibility with a relative standard deviation less than $2 \%$.

The observed values for $\mathrm{Cu}, \mathrm{Ag}, \mathrm{Pb}$, and $\mathrm{Sn}$ in NMIJ CRM 8203-a are summarized in Table 6 , along with the certified value for each element. It can be seen in Table 6 that the observed values were generally close to or in agreement with the certified values within their uncertainties. The difference between an observed value and a certified value could be attributed to the fact that the sample aliquot $(\mathrm{ca} .15 \mu \mathrm{g})$ used in a measurement by LA-ICP-MS were extremely less than that used for the analysis (not less than $0.2 \mathrm{~g}$, a whole tip) in the development of the CRM, approximately 1:13000. It can also be seen from
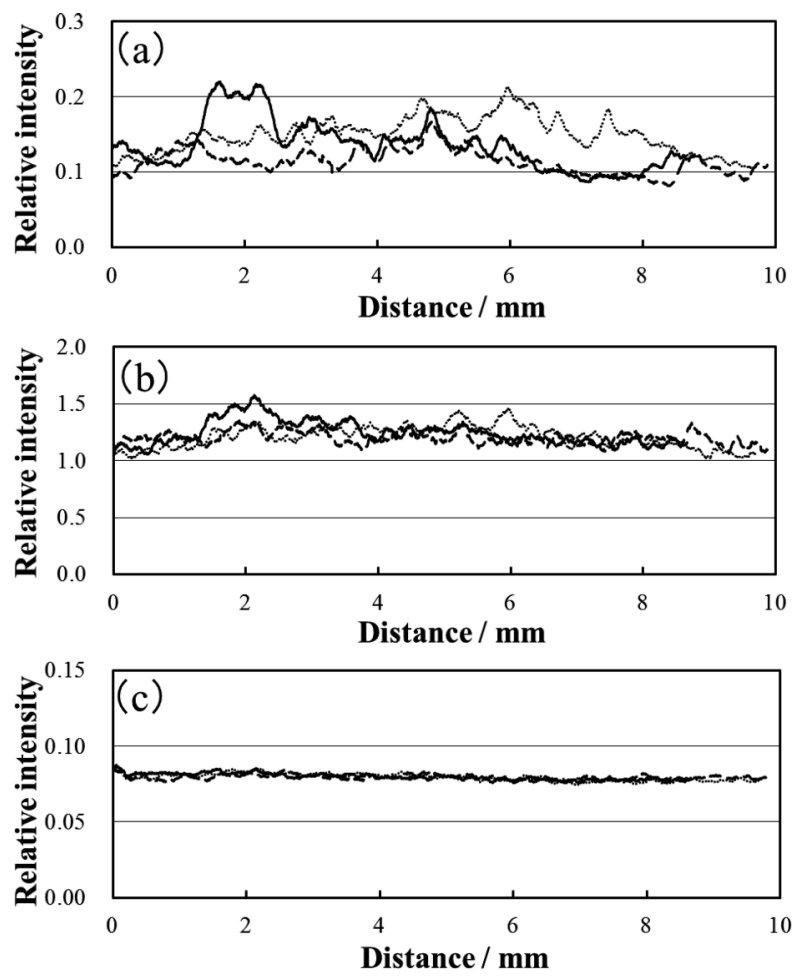

Fig. 4 Time profiles of signal intensity ratios obtained from three solder samples using the fs-LA-ICP-MS for (a) $\mathrm{Cu} / \mathrm{Sn}$, (b) $\mathrm{Ag} / \mathrm{Sn}$, and (c) $\mathrm{Pb} / \mathrm{Sn}$ ratios. The solid, dashed, and dotted lines represent the analysis number 1,2 , and 3 , respectively.

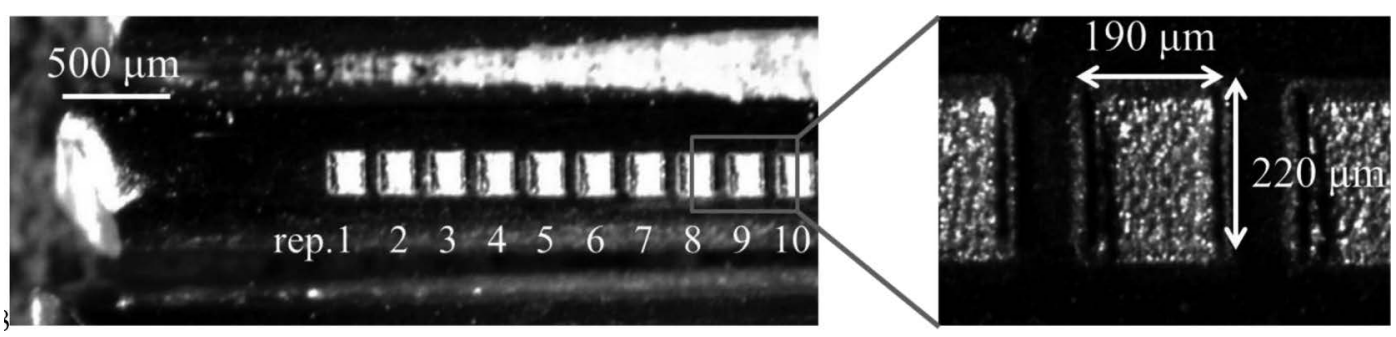

Fig. 5 Photographic images of ablation pit on surface of the solder sample. 
Table 4 Typical analytical results obtained by ns-LA-ICP-MS

\begin{tabular}{lcccc}
\hline \multirow{2}{*}{ Sample } & \multicolumn{4}{c}{ Signal intensity $/ \mathrm{CPS}$} \\
\cline { 2 - 5 } & ${ }^{63} \mathrm{Cu}$ & ${ }^{107} \mathrm{Ag}$ & ${ }^{12} \mathrm{Sn}$ & $108 \pm 3$ \\
\hline $\mathrm{HNO}_{3}$ & $548 \pm 93$ & $11 \pm 2$ & $386365 \pm 2064$ & $19 \pm 3$ \\
Standard-0 & $2909 \pm 548$ & $32 \pm 9$ & $377607 \pm 1519$ & $28 \pm 2$ \\
Standard-1 & $55898 \pm 2415$ & $51450 \pm 548$ & $363603 \pm 1105$ & $3882 \pm 25$ \\
Standard-2 & $106712 \pm 1942$ & $100955 \pm 451$ & $3631 \pm 47$ \\
Standard-3 & $207054 \pm 4082$ & $197685 \pm 1014$ & $362811 \pm 1387$ & $14134 \pm 84$ \\
NMIJ CRM 8203-a & $131432 \pm 5770$ & $120214 \pm 5345$ & $414873 \pm 16961$ & $9006 \pm 388$ \\
\hline
\end{tabular}

a. Mean \pm standard deviation, $n=10$.

Table 5 Elemental concentrations in the standard solutions and the solder sample

\begin{tabular}{crcccc}
\hline & \multicolumn{5}{c}{ Concentration } \\
\cline { 2 - 6 } Element & $\begin{array}{c}\text { Standard-0/ } \\
\text { ng g }^{-1}\end{array}$ & $\begin{array}{c}\text { Standard-1/ } \\
\text { ng g }^{-1}\end{array}$ & $\begin{array}{c}\text { Standard-2/ } \\
\text { ng g }^{-1}\end{array}$ & $\begin{array}{c}\text { Standard-3/ } \\
\text { ng g }^{-1}\end{array}$ & $\begin{array}{c}\text { NMIJ CRM } \\
8203-a / ~ \\
\mathrm{mg} \mathrm{g}^{-1}\end{array}$ \\
\hline $\mathrm{Cu}$ & 0 & 2.44 & 4.89 & 9.75 & 5.04 \\
$\mathrm{Ag}$ & 0 & 15.2 & 30.3 & 60.5 & 29.9 \\
$\mathrm{Sn}$ & 1014 & 1015 & 995 & 1008 & 965 \\
$\mathrm{~Pb}$ & 0 & 0.49 & 0.99 & 1.97 & 0.949 \\
\hline
\end{tabular}

Table 6 Analytical results of major and minor elements in NMIJ CRM 8203-a

\begin{tabular}{lllll}
\hline \multirow{3}{*}{ Value } & \multicolumn{4}{c}{ Concentration ${ }^{\mathrm{a}} / \mathrm{mg} \mathrm{g}^{-1}$} \\
\cline { 2 - 5 } & $\mathrm{Cu}$ & $\mathrm{Ag}$ & $\mathrm{Pb}$ & $\mathrm{Sn}$ \\
\hline
\end{tabular}

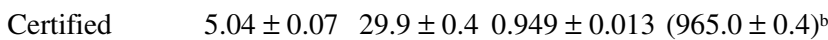
ns-LA-ICP-MS $4.58 \pm 0.21 \quad 29.5 \pm 0.7 \quad 1.137 \pm 0.061 \quad 965.9 \pm 1.0$ fs-LA-ICP-MS $4.94 \pm 0.23 \quad 26.0 \pm 0.6 \quad 0.925 \pm 0.018 \quad 969.1 \pm 0.7$

a. Mean \pm expanded uncertainty, $k=2$.

b. Information value calculated based on the concentration of $\mathrm{Cu}, \mathrm{Ag}$, and $\mathrm{Pb}$ in the sample.

Fig. 5 that only minute portions on the surface of a solder tip were analyzed in the present method. The quantity of sample used for the analysis in the development of the CRM was given in the certificate of the CRM, while the sample aliquot $(m)$ used in LA-ICP-MS could be roughly estimated based on Eq. (7)

$$
m=V \times d .
$$

In Eq. (7), $V$ is the sample volume ablated for 10 measurements, while the volume of each measurement could be calculated as $(c a .190 \times 220 \times 5 \mu \mathrm{m})$. The density $(d)$ of the solder sample was approximately $7.2 \mathrm{~g} \mathrm{~cm}^{-3}$.

The observed results were quite close to the certified values, indicating that the present method might serve as an effective method for rapid screening analysis of the elements in solder samples and so on. The present work provided a valid method for quantitative analysis of the elements in solid samples without relying on solid standards.

In the present work, it took approximately $40 \mathrm{~s}$ to measure a sample area of $(190 \times 200 \mu \mathrm{m})$. In addition, $20 \mathrm{~s}$ was enough for the washout between two measurements. Therefore, a single measurement could be finished in $1 \mathrm{~min}$. As a result, the analysis of 6 samples with 10 measurements for each sample could be achieved in $1 \mathrm{~h}$.

It is notable that only a minute quantity of the sample was subjected to the analysis, the results depended on the homogeneity of each element of interest in the sample. In order to obtain a result that represents the whole sample, multiple analyses of different parts of the sample are required.

\section{Conclusion}

In the present work, quantitative analysis of the elements in the solder sample by LA-ICP-MS was conducted with Sn-based matrix matched standard solutions for making calibration curves. By preventing the formation of a $\mathrm{AgCl}$ precipitate, a laboratory-made chloride-free Sn solution ensured the stability of the Ag concentration in Sn matrix matched standard solutions and the linearity of the calibration curve. The sample-surface irradiation over $12 \mathrm{~J} \mathrm{~cm}^{-2}$ in the laser ablation process effectively suppressed elemental fractionations, and permitted quantitative analysis of the elements in a solder sample by LA-ICP-MS. The results of quantitative analysis of $\mathrm{Ag}, \mathrm{Cu}, \mathrm{Pb}$, and $\mathrm{Sn}$ in NMIJ CRM 8203-a indicate that the present method was valid for the analysis of solder samples. The accuracy and the precision for the analytical results obtained by LA-ICP-MS should be evaluated taking into consideration the elemental homogeneity in a sample. The data obtained here clearly demonstrate that the combination of ns-LA or fs-LA ablation and multiple spot-laser ablation protocol can become a powerful approach for the elemental analysis of metallic materials. In comparison to the ns-LA instrument, the fs-LA instrument might suppress LA-induced elemental fraction under a relatively lower fluence condition.

\section{References}

1. R. E. Russo, X. L. Mao, H. C. Liu, J. Gonzalez, and S. S. Mao, Talanta, 2002, 57, 425.

2. D. Gunther, H. R. Kuhn, and M. Guillong, Geochim. Cosmochim. Acta, 2005, 69, A372.

3. J. S. Becker, A. Matusch, and B. Wu, Anal. Chim. Acta, 2014, 835,1 .

4. D. Pozebon, G. L. Scheffler, V. L. Dressler, and M. A. G. Nunes, J. Anal. At. Spectrom., 2014, 29, 2204.

5. N. Miliszkiewicz, S. Walas, and A. Tobiasz, J. Anal. At. Spectrom., 2015, 30, 327.

6. A. Limbeck, P. Galler, M. Bonta, G. Bauer, W. Nischkauer, and F. Vanhaecke, Anal. Bioanal. Chem., 2015, 407, 6593.

7. Y. S. Liu, Z. C. Hu, S. Gao, D. Gunther, J. Xu, C. G. Gao, and H. H. Chen, Chem. Geol., 2008, 257, 34. 
8. T. Iizuka and T. Hirata, Chem. Geol., 2005, 220, 121.

9. H. Obayashi, M. Tanaka, K. Hattori, S. Sakata, and T. Hirata, J. Anal. At. Spectrom., 2017, 32, 686.

10. P. Degryse and F. Vanhaecke, Elements, 2016, 12, 341.

11. J. Koch and D. Guenther, Appl. Spectrosc., 2011, 65, 155A.

12. D. Gunther, R. Frischknecht, C. A. Heinrich, and H.-J. Kahlert, J. Anal. At. Spectrom., 1997, 12, 939.

13. D. Gunther and B. Hattendorf, TrAC, Trends Anal. Chem., 2005, 24, 255.

14. D. Gunther, A. Audetat, R. Frischknecht, and C. A. Heinrich, J. Anal. At. Spectrom., 1998, 13, 263.

15. C. Chaleard, P. Mauchien, N. Andre, J. Uebbing, J. L. Lacour, and C. Geertsen, J. Anal. At. Spectrom., 1997, 12, 183.

16. M. Guillong and D. Gunther, J. Anal. At. Spectrom., 2002, $17,831$.

17. I. Kroslakova and D. Gunther, J. Anal. At. Spectrom., 2007, 22, 51.

18. P. K. Diwakar, S. S. Harilal, N. L. LaHaye, A. Hassanein, and P. Kulkarni, J. Anal. At. Spectrom., 2013, 28, 1420.

19. P. K. Diwakar, J. J. Gonzalez, S. S. Harilal, R. E. Russo, and A. Hassanein, J. Anal. At. Spectrom., 2014, 29, 339.
20. T. Luo, Y. Wang, Z. Hu, D. Guenther, Y. Liu, S. Gao, M. Li, and S. Hu, J. Anal. At. Spectrom., 2015, 30, 941.

21. M. Aramendía, M. Resano, and F. Vanhaecke, J. Anal. At. Spectrom., 2010, 25, 390.

22. C. O. Connor, B. L. Sharp, and P. Evans, J. Anal. At. Spectrom., 2006, 21, 556.

23. D. Gunther, H. Cousin, B. Magyar, and I. Leopold, J. Anal. At. Spectrom., 1997, 12, 165.

24. L. Halicz and D. Gunther, J. Anal. At. Spectrom., 2004, 19, 1539.

25. J. J. Leach, L. A. Allen, D. B. Aeschliman, and R. S. Houk, Anal. Chem., 1999, 71, 440.

26. C. Pickhardt, A. V. Izmer, M. V. Zoriy, D. Schaumlöffel, and J. Sabine Becker, Int. J. Mass Spectrom., 2006, 248, 136.

27. D. Pozebon, V. L. Dressler, M. Foster Mesko, A. Matusch, and J. Sabine Becker, J. Anal. At. Spectrom., 2010, 25, 1739.

28. Y. Zhu, Anal. Sci., 2016, 32, 1237.

29. T. D. Yokoyama, T. Suzuki, Y. Kon, and T. Hirata, Anal. Chem., 2011, 83, 8892. 